\title{
On the Nature of Semantic Constraints on Lexical Access
}

\author{
Andrea Weber • Matthew W. Crocker
}

Published online: 16 November 2011

(C) Springer Science+Business Media, LLC 2011

\begin{abstract}
We present two eye-tracking experiments that investigate lexical frequency and semantic context constraints in spoken-word recognition in German. In both experiments, the pivotal words were pairs of nouns overlapping at onset but varying in lexical frequency. In Experiment 1, German listeners showed an expected frequency bias towards high-frequency competitors (e.g., Blume, 'flower') when instructed to click on low-frequency targets (e.g., Bluse, 'blouse'). In Experiment 2, semantically constraining context increased the availability of appropriate low-frequency target words prior to word onset, but did not influence the availability of semantically inappropriate high-frequency competitors at the same time. Immediately after target word onset, however, the activation of high-frequency competitors was reduced in semantically constraining sentences, but still exceeded that of unrelated distractor words significantly. The results suggest that (1) semantic context acts to downgrade activation of inappropriate competitors rather than to exclude them from competition, and (2) semantic context influences spoken-word recognition, over and above anticipation of upcoming referents.
\end{abstract}

Keywords Spoken-word recognition $\cdot$ Semantic constraints $\cdot$ Eye-tracking $\cdot$ German

\section{Introduction}

In one episode of the famous Seinfeld sitcom, Jerry has trouble with his new "sentence finisher" date Lisi:

JERRY: Well, it was nice meeting you. I'm sure I'll see you-

LISI: At eight tomorrow?

\footnotetext{
A. Weber $(\varangle)$

Max Planck Institute for Psycholinguistics, Wundtlaan 1, 6525 XD Nijmegen, The Netherlands e-mail: andrea.weber@mpi.nl
} 
JERRY: Actually, that's-

LISI: What you were thinking.

JERRY: Right.

While Jerry is clearly irritated by this habit of Lisi to try to end his sentences for him (and quickly ends the relationship), thinking ahead how a sentence might be continued could potentially be helpful for understanding spoken language. In particular, it might ease the recognition of words in a sentence if only words are considered that semantically fit the context. The present study set out to examine the nature of such semantic context effects on spoken-word recognition.

Recognizing spoken words involves the automatic activation of multiple candidate words that match the incoming acoustic signal. Thus, when hearing the word carpet, words that overlap at onset, like carbine, carton and carnival, are initially activated in parallel with what eventually proves to be the correct hypothesis (e.g., Allopenna et al. 1998; Zwitserlood 1989; Zwitserlood and Schriefers 1995). Activated word candidates compete with each other as long as they are consistent with the speech signal. Thus, upon encountering the $/ \mathrm{p} /$ of carpet, neither carbine, carton or carnival will compete any longer due to a mismatch with the acoustic information. The best matching candidate wins the competition process and is recognized (e.g., Luce and Pisoni 1998; Vitevitch 2002; Vitevitch and Luce 1998, 1999). This activation-competition process is assumed by most current models of spoken-word recognition such as TRACE (McClelland and Elman 1986), Shortlist B (Norris and McQueen 2008), DCM (Gaskell and Marslen-Wilson 1997), and PARSYN (Luce et al. 2000), with the details of the process still being debated.

However, words are usually not encountered in isolation but in the context of other words, and it has hence been a long-standing debate in psycholinguistic research, how the context of an utterance influences spoken-word recognition. In particular, the debate has concentrated on if and when semantic constraints in sentential context act to select a set of contextually appropriate candidate words. Thus, is carbine still competing with carpet in the context of "The cleaner is vacuuming the car..."? If no, when exactly did context exert its influence? If yes, how strong is carbine activated in such a context? The research reported in this article addresses these issues by examining the competition process of phonologically related words in an eye-tracking study with neutral and semantically constraining verbs. Based on our results, we will argue that semantic context can influence processing in two ways: (1) it can make appropriate conceptual representations available before they are encountered in the speech signal, and independently thereof (2) it can influence the activation of phonological competitors once the acoustic signal of a word is being processed. Furthermore, even though the activation of semantically ill-fitting phonological competitors is reduced, they still compete for recognition. While our findings are largely consistent with recent eye-tracking literature, they provide new insights about the nature of context effects in spoken-word recognition, and they demonstrate the influence of frequency in sentence context for phonologically overlapping words rather than for semantically ambiguous words.

Classic evidence suggesting that contextual information does not constrain initial lexical activation comes from cross-modal priming studies. In particular, Zwitserlood (1989) showed that as long as gated auditory primes are consistent with two Dutch words (e.g., /kap/ in kapitein, 'captain', and kapitaal, 'capital'), visual target words that are semantically related to either kapitein or kapitaal are primed, even when sentential context biases strongly against them. While for an early probe position, priming effects were comparable for target words with an appropriate and an inappropriate meaning, priming effects became soon stronger for the appropriate meaning, even before the signal provided disambiguating information. 
This finding suggests that contextual information is not used to determine which words are considered for recognition, but is used rapidly thereafter to select among the set of activated candidates.

Other studies in support of the view that initial lexical activation is driven strictly by the acoustic input for the word, employed words with multiple meanings. In a seminal crossmodal associative priming study, Swinney (1979) has shown that both meanings of fully ambiguous words (e.g., spying device and insect meaning of bug) are activated at word offset, regardless of a semantic bias in the preceding context for one meaning or the other, but shortly after word offset only the semantically appropriate meaning was still activated. Many other studies with a variety of experimental paradigms also found that semantic and syntactic constraints did not inhibit the early activation of contextually inappropriate meanings for fully ambiguous words (Blutner and Sommer 1988; Lucas 1987; Oden and Spira 1983; Onifer and Swinney 1981; Seidenberg et al. 1982; Tanenhaus and Donenwerth-Nolan 1984; Tanenhaus et al. 1979; Whitney et al. 1985). Only when an ambiguous word has a dominant and a subordinate meaning, several semantic priming studies have found little or no priming for the subordinate meaning in contexts that bias strongly towards the dominant meaning but not the reverse (e.g., Moss and Marslen-Wilson 1993; Simpson 1981; Tabossi 1988; Tabossi et al. 1987; Tabossi and Zardon 1993). Thus, the subordinate red wine meaning of port is not activated in the context of "the ships that were in the port, ...", but the dominant haven meaning of port is activated in the context of "the host took a bottle of Barolo, instead of port ..." (Tabossi et al. 1987). Comparable effects have been found in numerous reading studies: when sentence context supports the subordinate meaning of an ambiguous word, readers fixate longer on ambiguous words than on unambiguous controls matched for frequency, presumably reflecting a competition process between the two meanings (e.g., Duffy et al. 1988; Kambe et al. 2001; Sereno et al. 1992).

A potential disadvantage for defining the moment of context effects in the studies described above is the fact that both cross-modal priming and reading measures are taken after at least part of the ambiguous word was heard. It is feasible though that semantic context effects happen at the earliest moments of lexical access or even before that. Monitoring participants' eye movements to displayed objects while they are listening to sentences, provides a continuous measure of how listeners interpret the speech stream and offers therefore the unique possibility to observe semantic context effects as the sentence unfolds. Huettig and Altmann $(2004,2007)$ have employed the eye-tracking methodology to investigate meaning activation of biased ambiguous words like pen in neutral sentence contexts and in sentence contexts that biased towards the subordinate meaning of the ambiguous word. They have found that in neutral contexts, at homonym onset, participants fixated pictures depicting the dominant meaning (writing pen) or the subordinate meaning (enclosure pen) as often as they fixated pictures of unrelated distractors; at homonym offset, however, both dominant and subordinate pictures were fixated significantly more. In biasing sentences like "The welder locked up carefully, but then checked the pen...", participants preferred to look at the subordinate picture already at homonym onset and they continued to do so throughout the word. The dominant picture did not attract more looks at homonym onset, but did by the time the end of the homonym was reached. Huettig and Altmann (2007) concluded from their results that perceptual representations of the contextually inappropriate dominant referent were accessed even though the contextually more appropriate subordinate referent was also displayed (and activated).

While Huettig and Altmann (2007) did not focus on the fact that conceptual representations of the subordinate meaning were activated in their study already at homonym onset, anticipation of contextually appropriate referents was the focus of an influential eye-tracking 
study by Altmann and Kamide (1999). They have shown that selective restrictions associated with verbs can be used to predict the semantic class of forthcoming themes. In their study, English participants fixated a displayed edible object more than inedible objects upon hearing "The boy will eat ...", even prior to the post-verbal expression "the cake"; in sentences with less constraining verbs such as "The boy will move ...", edible and inedible objects were fixated equally often. In subsequent studies, Kamide et al. (2003) have shown that information from the verb was combined with information about its grammatical subject to further constrain prediction of the verb's likely argument.

While these eye-tracking studies convincingly showed anticipatory effects of subject and verb information on the activation of conceptual representations, they did not directly test the influence of selective verb restrictions on the activation of phonologically related representations. The activation of such form-based representations is, however, at the heart of most models of spoken-word recognition. Spoken-word recognition involves the mapping of the incoming speech signal onto matching phonological representations; activated phonological representations in turn activate conceptual representations, and together they can yield the meaning of an utterance. A distinction between phonological and conceptual representations is generally made and is empirically supported, for instance, by differences in form-based and meaning-based priming with the same set of primes (Gaskell and Marslen-Wilson 2002; Norris et al. 2006) or differences in the time course of activation for phonological and semantic competitors (Huettig and McQueen 2007). Dahan and Tanenhaus (2004) have taken up this issue in a Dutch eye-tracking study in which they investigated the effect of selective verb restrictions on the subsequent activation of phonologically related nouns. In their study, Dahan and Tanenhaus displayed four object pictures on a screen: the Dutch names of two of them overlapped phonemically at onset (e.g., bok, 'goat', and bot, 'bone'), a third name was semantically related to one of the phonemically overlapping names (e.g., spin, 'spider' which is related to bok, 'goat'), while the fourth name was completely unrelated (e.g., eiland, 'island'). In accompanying sentences, the main verb either preceded the subject argument bok-and thus semantically constrained which picture name(s) could be the subject argument — or not (e.g., "Nog nooit klom een bok zo hoog", 'Never before climbed a goat so high', or "Nog nooit is een bok zo hoog geklommen", 'Never before has a goat climbed so high'); the participants' task was to indicate with a mouse-click which of the four displayed objects was mentioned in the sentence. Dahan and Tanenhaus (2004) found that both bok and bot attracted more looks than the other pictures when the onset of the target word bok was heard before the main verb, reflecting simultaneous activation of the two phonologically related words. When the semantically constraining main verb occurred before the subject, however, the semantically appropriate bok attracted more looks than it had in the neutral context, whereas the semantically inappropriate bot attracted fewer looks. Because their effects emerged at the earliest moments of lexical processing, Dahan and Tanenhaus (2004) interpreted them as evidence for an immediate rather than delayed influence of semantic sentence context on the recognition of spoken words. In a study on the use of common ground Barr (2008) has found comparable effects. In one of the experiments, he compared the influence of pragmatic and semantic constraints and found that phonological competitors were no longer fixated more than distractor objects when a preceding verb made them semantically inappropriate.

The Dahan and Tanenhaus (2004) data and the Barr (2008) data did not allow conclusions about the underlying mechanism of the context effect. Does sentence context facilitate the recognition of semantically appropriate words or does it inhibit the recognition of inappropriate words, or maybe both? The probability to fixate an object on the screen is not independent of the probability to fixate other displayed objects (one can only look at one thing at a time); 
fewer looks to the inappropriate bot could therefore either be due to inhibition or just mirror increased attention for the appropriate bok. Dahan and Tanenhaus furthermore found no significant difference in looks to inappropriate competitor bot versus looks to distractors. Does this mean that the competitor bot was no longer considered a candidate during the recognition of bok? A lack of a difference between looks to competitor and distractor is usually interpreted in eye-tracking studies as a lack of activation of the competitor (e.g., Dahan et al. 2000; Weber and Cutler 2004). In contrast to Dahan and Tanenhaus (2004), Huettig and Altmann (2007) did find significantly more looks to contextually inappropriate object pictures than to distractors. In their study, however, the inappropriate picture depicted the more frequent meaning of a homonym. It is quite possible therefore that Dahan and Tanenhaus (2004) did not find significant activation of inappropriate phonological competitors because they were not higher in lexical frequency than their appropriate targets were.

The aim of the eye-tracking studies reported here was two-fold. Firstly, we wanted to investigate the effect of selective verb information on phonologically related words that differ in lexical frequency. Words that occur frequently in a language are recognized faster and more easily than words that occur rarely; this word frequency effect has repeatedly been demonstrated not only with different reaction time paradigms like auditory lexical decision (e.g., Connine et al. 1990; Dupoux and Mehler 1990), rhyme monitoring (McQueen 1993), and priming (Marslen-Wilson 1990), but also with eye-tracking (Dahan et al. 2001; Dahan and Gaskell 2007). With respect to eye movements, it has been shown that phonological competitor objects with high lexical frequency are fixated more than phonological competitor objects with low lexical frequency. If selective verb restrictions are exclusive in nature, then we should find no significant activation of semantically inappropriate competitors even when they are higher in lexical frequency than the appropriate targets. If they are not exclusive in nature, then semantically inappropriate competitors should be activated and should be looked at more than unrelated distractors. Secondly, by re-examining the time course of semantic context effects, we want to disentangle anticipatory eye movements to semantically fitting target pictures, from an influence of context on the competition process when the target names are encountered in the speech stream.

To this end, we conducted two eye-tracking experiments in German. The first experiment set out to affirm lexical frequency effects for our German materials. We selected pairs of words with overlapping onset such as Bluse, 'blouse', and Blume, 'flower', but varying frequency (Bluse has a low frequency in German and Blume has a high frequency). It was predicted that both words should be considered as candidates (i.e., should be fixated more than objects with unrelated names) upon hearing their respective onsets, but high frequency Blume should be looked at more when being instructed to click on low frequency Bluse than low frequency Bluse should be looked at when being instructed to click on high frequency Blume. It was important to establish a frequency effect, in order to be able to correctly interpret the influence of semantic constraints in the second experiment. In the second experiment, low frequency Bluse was presented in a sentence context in which the verb either did or did not make highfrequency Blume an inappropriate argument. In an unrestrictive context (e.g., Die Frau findet, 'The woman finds'), high frequency Blume should be a stronger candidate for recognition than low frequency Bluse due to its frequency advantage. If sentential restrictions indeed fully eliminate activation of inappropriate candidate words, high frequency Blume should no longer be fixated in the context of Die Frau bügelt, 'The woman irons', regardless of its frequency advantage. If, on the other hand, sentential restrictions only act to downgrade the activation of inappropriate word candidates, Blume should still be a candidate for recognition in restrictive contexts, albeit a weaker candidate than in unrestrictive contexts. Furthermore, if anticipation of appropriate target referents occurred separately of a modulation of 
competitor activation during spoken-word recognition, this would be strong evidence for the lexical competition process itself being semantically informed.

\section{Experiment 1}

Method

Participants

Twenty students from Saarland University, all native speakers of German, were paid to take part in the experiment.

\section{Materials}

We selected 20 pairs of German words which all were the names of picturable objects. Within each pair of words, names overlapped phonemically at onset and differed in lexical frequency (e.g., high frequency Blume, 'flower', and low frequency Bluse, 'blouse'). According to CELEX (Baayen et al. 1993), high frequency lemmas had an average frequency of 44.6 per million (1.5 log per million) compared to 3.6 (0.4 log per million) for low frequency lemmas $(F[1,19]=9.88, p<.005)$. The two names of each pair were matched for syllable number (with the exception of Thermometer-Telefon which differed in one syllable) and overlapped at onset for an average of 2.5 phonemes. Given the lexical frequency constraints and the semantic constraints in Experiment 2, however, the extent of phonemic overlap could not be equated across pairs. Coloured line drawings were selected for both words in a pair, and were displayed together with the drawing of a human character and a distractor object with a name that was semantically and phonologically unrelated to the other drawings in the display (e.g., Wolke, 'cloud', see Fig. 1). Even though previous research has shown that the probability of fixating unrelated distractors does not vary with lexical frequency (Dahan et al. 2001), we wanted to ensure the general accessibility of our distractors and selected distractor objects with frequencies that were similar to our high frequency objects (47.0 per million on average, $1.07 \mathrm{log}$ per million). The human character corresponded to the agent of the accompanying sentence in Experiment 2 and either was a man, a woman, a girl, or a boy. Positions of the picture types were evenly distributed across the four fixed positions. To ensure that the object drawings evoked the intended nouns in participants, a picture-naming task was administered. Responses from an independent group of 12 German speakers were on average $87.3 \%$ correct, with the highest scores for distractor objects $(96.3 \%)$, followed by $88.8 \%$ for high-frequency objects, and $78.7 \%$ for low-frequency objects $(F[2,57]=7.82, p<.01)$; a pair-wise comparison between low frequency and high frequency objects was not significant $(F[1,19]=4.36, p>.05)$. When synonymous names were included as correct responses, average scores were $96.3 \%$ for distractor objects, $92.7 \%$ for high frequency objects, and $89.7 \%$ for low frequency objects, $(F[2,57]=4.14, p>.05)$. Synonymous responses were included because they indicate that participants identified objects correctly; also Weber and Melinger (2008) have shown in an eye-tracking study with synonyms, that both the dominant and the subordinate name of a synonymous competitor are indeed considered for word recognition.

There were an additional 30 four-picture displays that were used in filler trials, and a further two such displays used in preliminary practice trials. Some of these displays contained pairs of pictures with phonologically or semantically related names (e.g., a palm 

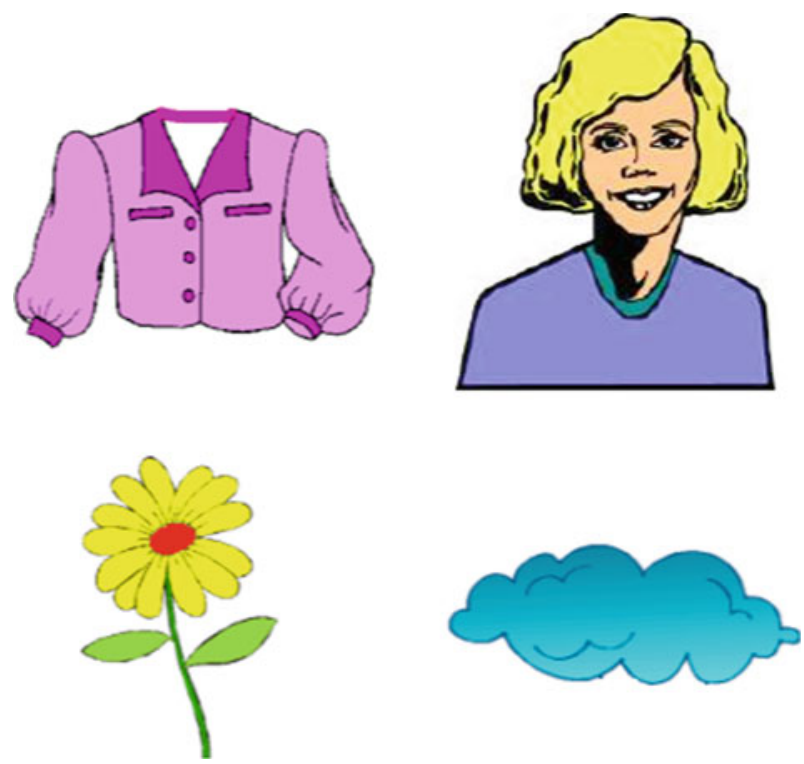

Fig. 1 Example of stimulus display

tree and a palace, or a banana and an apple). In others, there was no phonological or semantic relationship between the four names. No pictures were used in more than one trial.

Spoken instructions to click on one of the object pictures accompanied the displays. The instructions referred to either the high-frequency object (e.g., Klicke auf die Blume, 'click on the flower') or to the low-frequency object (e.g., Klicke auf die Bluse, 'click on the blouse'). We recorded a female native speaker of German onto Minidisk in a quiet room, sampling at $44.1 \mathrm{kHz}$. The recordings were then down-sampled to $22 \mathrm{kHz}$ and stored on a computer. Gender marked articles in the German instructions could not bias towards a particular object, since all objects in a display shared gender. Durations of the preceding context (e.g., Klicke auf die) were measured using Praat; the average duration of the preceding context was $1283 \mathrm{~ms}$ in trials with high-frequency targets and $1261 \mathrm{~ms}$ in trials with low-frequency targets. In addition, the duration of the putative overlap of a target word and its competitor (e.g., the duration of $/ \mathrm{blu} /$ in Blume and Bluse) was measured. The average duration of this portion was $326 \mathrm{~ms}$ for high-frequency targets and $347 \mathrm{~ms}$ for low-frequency targets. The average duration of the whole target word was $676 \mathrm{~ms}$ for high frequency words and $685 \mathrm{~ms}$ for low frequency words.

Two experimental lists were constructed. Each version began with the two practice trials and further contained the 20 experimental trials and all 30 filler trials, in pseudo-random order such that before each experimental trial there was at least one filler trial. Experimental trials appeared once in a given list, counterbalancing between lists whether the high-frequency object or the low-frequency object was the target in the auditory instructions.

\section{Procedure}

At the beginning of a session, participants were informed that they would hear instructions to mouse-click on one of the pictured objects on the screen. They were then seated approximately 
$60 \mathrm{~cm}$ in front of a monitor, and their eye movements were monitored with an SMI EyeLink I eye tracker; onset and offset times and the spatial coordinates of fixations and saccades were recorded $(250 \mathrm{~Hz}$ sampling rate). After calibration of the eye tracker, each participant was presented with the trials from one of the trial lists. Images (each fitting in $200 \times 200$ pixels space) were presented in colour on a $21^{\prime \prime}$ monitor at a resolution of $1024 \times 768$ pixels. The edges of the pictures on the screen were approximately $5 \mathrm{~cm}$ apart (corresponding to a visual angel of $4.7^{\circ}$ ). Sentences were presented auditorily over headphones and started $1 \mathrm{~s}$ after the appearance of an image on the screen. Between trials, participants were told to fixate a small circle in the centre of the screen for an automatic drift correction.

\section{Results and Discussion}

For the analysis, custom-made graphical software was used to display locations of participants' fixations as dots superimposed on trial displays. Fixations were coded as pertaining to the agent, the target, the competitor, or the distractor. Fixations that did not fall on any of the displayed objects were coded as looks to the background. Blinks were added to previous fixations; saccade times were not added to fixation times. One experimental trial was excluded from the analyses because the mouse click occurred on the wrong object. For the remaining 399 trials, we computed proportions of fixations by adding the number of trials for each participant and each item that each of the four picture types was fixated during successive $10 \mathrm{~ms}$ time frames. The sum for each alternative picture type was then divided by the total sum of all fixations during the interval. Figure 2 displays the fixation proportions (averaged over participants and in $40 \mathrm{~ms}$ steps) to the target, the competitor, and the distractor from 0 to $1000 \mathrm{~ms}$ after target-word onset for trials with high-frequency competitors (Fig. 2a) and low-frequency competitors, respectively (Fig. 2b). Even though the agent is functionally a second distractor in Experiment 1, we refrained from averaging its fixation proportions with the unrelated distractor, for comparability with Experiment 2, in which the agent is actively named during the experiment and does not serve the same function as the unrelated distractor.

As can be seen in Fig. 2a, fixation proportions to the different picture types started to diverge after $200 \mathrm{~ms}$. It typically takes about $200 \mathrm{~ms}$ to program and initiate an eye movement (see e.g., Hallet 1986; Matin et al. 1993); $200 \mathrm{~ms}$ after target word onset is thus the starting point for fixations that are driven by the acoustic input from the target. While fixation proportions to the high-frequency competitor Blume started to increase around $200 \mathrm{~ms}$, fixation proportions to the distractor started to decrease at this point. Fixation proportions to the competitor started to drop again after $500 \mathrm{~ms}$, which corresponds quite well to the point in time when acoustic information distinguishes target and competitor ( $347 \mathrm{~ms}$ putative overlap plus $200 \mathrm{~ms}$ delay in eye movements), but the proportions of competitor fixations remained higher than distractor fixations at least until $900 \mathrm{~ms}$ after target word onset. More looks to competitor objects than to distractor objects shows that German listeners considered the competitor as a possible candidate for word recognition. This competition effect was particularly strong, and produced even more looks to the high-frequency competitor than to the low-frequency target object between 200 and 600 ms. Note that although Dahan and Gaskell (2007) tested the influence of lexical frequency on lexical activation in similar setup, they did not find any evidence of the high-frequency competitor outperforming the low-frequency target initially. A possible reason for this may be a smaller difference in lexical frequency between target and competitor than in the present study (Dahan and Gaskell: 1.7 for high-frequency objects and 0.8 for low-frequency objects).

When the competitor was of low lexical frequency (Fig. 2b), fixation proportions to the different picture types also started to diverge after $200 \mathrm{~ms}$. As in Fig. 2a, competitor objects 

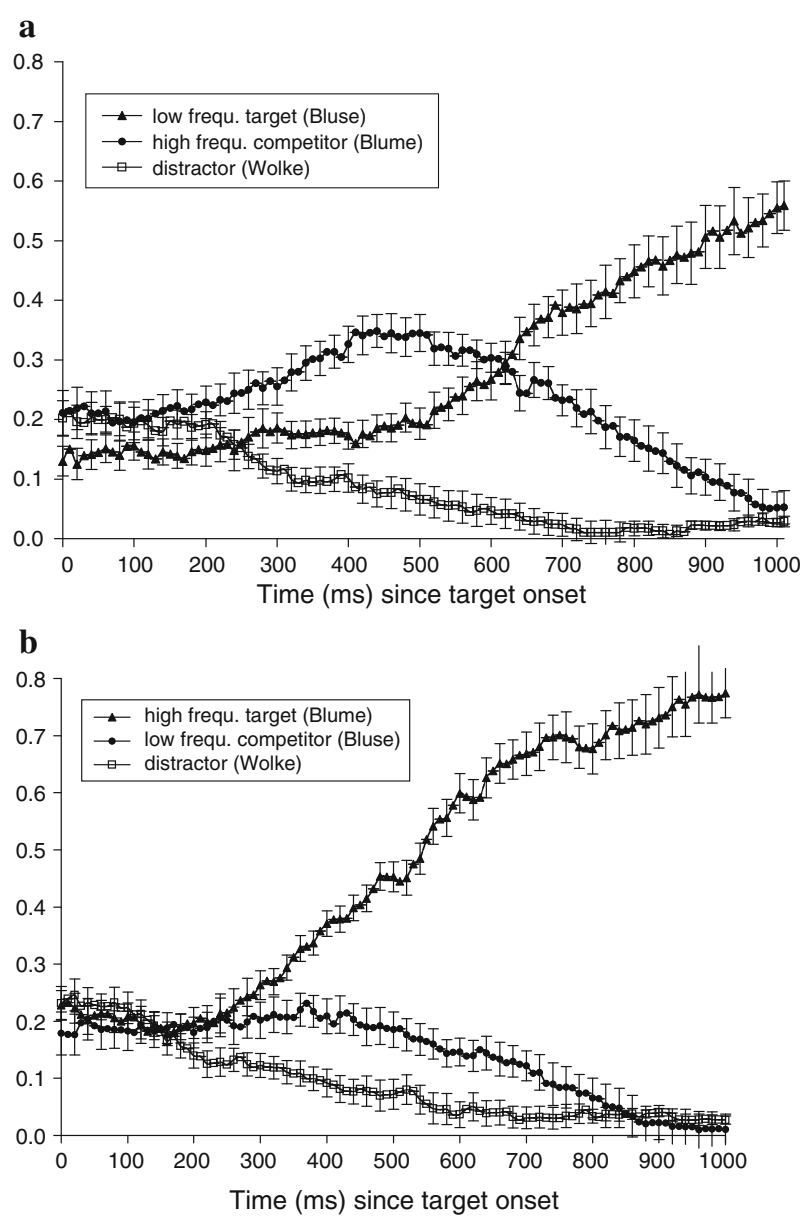

Fig. 2 a Fixation proportions over time to target, competitor, and distractor for trials with high-frequency competitors. Error bars represent standard errors of the mean. b Fixation proportions over time to target, competitor, and distractor for trials with low-frequency competitors. Error bars represent standard errors of the mean

were fixated more than distractor objects between 200 and $800 \mathrm{~ms}$; thus low-frequency competitors too induced a competition effect that was observable in eye movements, but the competition effect was smaller for low-frequency competitors in Fig. $2 b$ than for high-frequency competitors in Fig. 2a. Accordingly, targets with high-frequency names (Fig. 2b) were fixated more throughout a trial than targets with low-frequency names (Fig. 2a). It is possible that low-frequency targets received in part fewer looks because the pre-test showed somewhat more ambiguity in naming for these items. The clicking responses show, however, that participants had no difficulties to identify intended low-frequency targets, and eventually (between 1100 and $1300 \mathrm{~ms}$ ) low-frequency and high-frequency targets did reach comparable maximum values $(71 \%$ for low-frequency targets and $80 \%$ for high-frequency targets). Furthermore, correlation analyses revealed no significant correlation between looks to low-frequency targets and naming scores $(r(18)=-0.07, p>.7)$. This makes it unlikely 
that fewer looks to low-frequency target reflect difficulties of listeners to identify objects associated with low-frequency names.

To statistically confirm this pattern, we first arcsine-transformed the mean fixation proportions $(y=\operatorname{arcsine}(x))$, and then compared the magnitude of the competition effect for trials with high-frequency competitors and low-frequency competitors. To this end, we subtracted (arcsine-transformed) fixation proportions to the competitor from fixation proportions to the distractor for two time windows; one extending from 0 to $200 \mathrm{~ms}$ after target onset, and the other from 200 and $900 \mathrm{~ms}$. The later time window of $700 \mathrm{~ms}$ corresponded approximately to the average duration of target words $(676 \mathrm{~ms}$ for high frequency targets and $685 \mathrm{~ms}$ for low frequency targets). Between 0 and $200 \mathrm{~ms}$, when fixations are not yet driven by acoustic input from the target word, there was no notable difference between competitor and distractor fixations (2\% difference, both for trials with high-frequency competitors and for trials with low-frequency competitors). Between 200 and $900 \mathrm{~ms}$, the advantage for the competitor was on average $18 \%$ for trials with high-frequency competitors compared to $9 \%$ for trials with lowfrequency competitors. This difference was significant in a one-way ANOVA by subjects and marginally significant by items $\left(F_{1}[1,19]=7.89, p<.02 ; F_{2}[1,19]=3.41, p<.08\right)$. After removing the item pair with the smallest variation in lexical frequency (i.e., FlascheFlagge $)$, the difference was fully significant by items $\left(F_{2}[1,18]=4.78, p<.05\right)$; for a shorter analysis window that reflects the duration of putative overlap (i.e., 200-600 ms), an ANOVA with the complete set of items was also significant by subjects and items $\left(F_{1}[1,19]=\right.$ $\left.5.51, p<.03 ; F_{2}[1,19]=4.48, p<.05\right)$. This suggests that high-frequency competitors like Blume exerted a stronger competition effect than low-frequency competitors such as Bluse in German listeners. Previous research has shown that not only lexical frequency but also age of acquisition influences lexical processing (e.g., Bonin et al. 2004; Fiebach et al. 2003). We did not collect data on the age of acquisition for our materials, but a significant correlation between lexical frequency and fixation proportions $(r(38)=.38, p<.02)$ suggests that our findings are indeed reflecting lexical frequency effects. This finding furthermore is in line with previous evidence for lexical frequency effects in eye movements for English and Dutch listeners (Dahan and Gaskell 2007; Dahan et al. 2001). In these studies too, high-frequency objects were activated more strongly (i.e. looked at more often) than lowfrequency objects.

After having established in Experiment 1 that our experimental items induce lexical frequency effects for German listeners, we set out in Experiment 2 to examine the activation of high-frequency words in semantically constraining sentences. For this purpose, we presented trials with low-frequency targets, like Bluse, and high-frequency competitors, like Blume, to German listeners, while they were hearing sentences in which the verb either did or did not render the competitor a semantically unlikely object (e.g., Blume, 'flower', is a plausible object in the context of Die Frau findet die..., 'The woman finds the. ..', but an implausible object in the context of Die Frau bügelt die..., 'The woman irons the. .'). Two hypotheses were made: (1) If semantically constraining verbs exclude implausible competitors as possible word candidates, then we should observe no competition effect for these high-frequency competitors following a constraining verb. If on the other hand, the impact of semantically constraining verbs does not lead to a complete exclusion of implausible candidates but rather to a downgrading of the activation of these candidates, then we should observe some competition for high-frequency competitors even when they are semantically implausible. (2) If semantic context only helps to pre-select upcoming referents in a sentence, then competitor activation should be just a reflection of that. If on the other hand, the competition process during spoken-word recognition is informed by context, then we should find competitor activation independently of anticipatory eye movements. 


\title{
Experiment 2
}

\author{
Method
}

\section{Participants}

Forty-eight native speakers of German, students at the Saarland University, were paid to take part in the experiment. None of the participants had taken part in Experiment 1. As before, all had normal or corrected-to-normal vision and normal hearing.

\section{Materials}

The materials were as in Experiment 1, except that the auditory sentences accompanying the experimental displays were semantically richer in content and always referred to the low-frequency object (e.g., Bluse). These sentences started with a subject noun phrase (NP), followed either by a semantically non-restrictive or restrictive main verb in present tense, and a second noun phrase referring to the low-frequency object on the screen (e.g., Die Frau findet/bügelt die Bluse, 'The woman finds/irons the blouse'; see "Appendix"). Subject NPs were chosen so as to not bias strongly against particular object NPs (i.e., a blouse and a flower are typical for a woman to handle) nor did the gender marking of articles in the second NP exclude upcoming arguments since objects in a display always shared gender. The restrictive verb, however, strongly biased towards the low-frequency target and against the high-frequency competitor (i.e., ironing a blouse is very plausible, whereas ironing a flower is not). For German verbs, we only know of one large-scale association database (Schulte im Walde 2008). For those restrictive verbs of the present study that were tested in this database, associations with the low-frequency target were weak (i.e., frequency of occurrence $=1$ ) or non-existing. To ensure that both the low-frequency target and the high-frequency competitor were equally plausible arguments for sentences with unrestricting verbs, a rating study was administered to 8 participants. Participants were asked to judge how plausible the event described in the sentences was on a scale from 0 (very implausible) to 6 (very plausible). Since all events described in the unrestrictive sentences were quite plausible, 20 filler sentences varying in plausibility were added to the rating test. On average, unrestrictive sentences with low-frequency targets were rated with 4.8 (e.g., the woman finds the blouse), compared to 4.9 for unrestrictive sentences with high-frequency competitors (e.g., the woman finds the flower, both $F s<1$ ). In a second rating study, an additional 10 participants rated the plausibility of restrictive sentences with the low-frequency target or with the high-frequency competitor (again 20 filler sentences with varying plausibility were added to the list of restrictive sentences). On average, participants rated sentences with high-frequency competitors with 1.0 (e.g., the woman irons the flower) and sentences with low-frequency targets with 5.1 (e.g., the woman irons the blouse). Thus, overall high-frequency competitors were clearly implausible referents in our restrictive sentences. Note, however, that two of the sentences with competitors were rated considerably more plausible than the other sentences with competitors (ranging from 0.0 to 1.6): Lampe 'lamp' with 4.4. and Schublade 'drawer' with 4.2. For these sentences too, however, the corresponding sentences with high-frequency targets were still rated higher in plausibility (4.8 and 5.8 respectively).

The displays were the same as in Experiment 1, although in Experiment 2, for half of the trials we replaced the phonological competitor with a second unrelated distractor. While Altmann and Kamide (1999) had found clear anticipatory eye movements to upcoming 
semantically appropriate referents in a display without further phonological competitors, anticipation had been less pronounced in the Dahan and Tanenhaus study (2004) that did contain phonological competitors. The purpose of comparing trials with and without phonological competitors in Experiment 2, was to see whether the make up of the display influences the strength of anticipatory eye movements to appropriate objects. The results showed, however, that anticipatory eye movements were not influenced by the absence or presence of phonological competitor objects in Experiment 2. Since the focus of this article is rather on the comparison between target and competitor objects, the results for trials without phonological competitors will not be reported any further.

Sentences accompanying the filler displays in Experiment 2 employed a variety of syntactic structures and varied in plausibility. All sentences were again recorded by a native speaker of German onto Minidisk in a quiet room, sampling at $44.1 \mathrm{kHz}$. The recordings were then down-sampled to $22 \mathrm{kHz}$ and stored on a computer. Durations of the preceding context (e.g., Die Frau findet/bügelt die) were on average $1013 \mathrm{~ms}$ for sentences with unrestrictive verbs and $1026 \mathrm{~ms}$ for sentences with restrictive verbs. The duration of putative overlap between low-frequency target words and their high-frequency competitors (e.g., the duration of /blu/ in Bluse) was $265 \mathrm{~ms}$ in restrictive sentences and $274 \mathrm{~ms}$ in unrestrictive sentences. The average duration of the whole target words was $550 \mathrm{~ms}$ in restrictive sentences and $564 \mathrm{~ms}$ in unrestrictive sentences. Note that word durations were approximately $130 \mathrm{~ms}$ shorter in Experiment 2 than in Experiment 1; the difference was caused by a slightly faster speaking rate in Experiment 2.

All trials were included in two experimental lists, counterbalancing between versions whether accompanying sentences contained a semantically restrictive or unrestrictive verb.

\section{Procedure}

Twenty-four German participants took part in the experiment. The procedure was very similar to the procedure in Experiment 1. Only this time, participants were informed that they would hear sentences that refer to some of the pictures on the screen, and that their task was to just listen carefully while inspecting the screen.

\section{Results and Discussion}

Figure $3 \mathrm{a}$ and $\mathrm{b}$, display fixation proportions to the low-frequency target, the high-frequency competitor, and the distractor from 0 to $1000 \mathrm{~ms}$ after target-word onset, for sentences with unrestrictive verbs (Fig. 3a) and for sentences with restrictive verbs (Fig. 3b) respectively.

When the verb was semantically unrestrictive (Fig. 3a), no early preference for the target was observed. While looks to the competitor started to increase around $250 \mathrm{~ms}$, looks to the distractor started to decline at the same time. Around $450 \mathrm{~ms}$ (the approximate end of overlap between target and competitor; $274 \mathrm{~ms}$ putative overlap plus $200 \mathrm{~ms}$ delay in eye movements), looks to the competitor started to decline again, but remained higher than the distractor until $800 \mathrm{~ms}$ after target onset. Thus, in the absence of a constraining verb, the high-frequency competitor Blume 'flower' was considered as a strong possible word candidate. As in Experiment 1, high-frequency competitors were initially fixated more than low-frequency targets (Fig. 2a), in accordance with their lexical frequency distribution. Thus, a reflection of lexical frequency, in the sense of more looks to high-frequency competitors than low-frequency targets, was also observed in Experiment 2 with semantically richer sentences than in Experiment 1. 

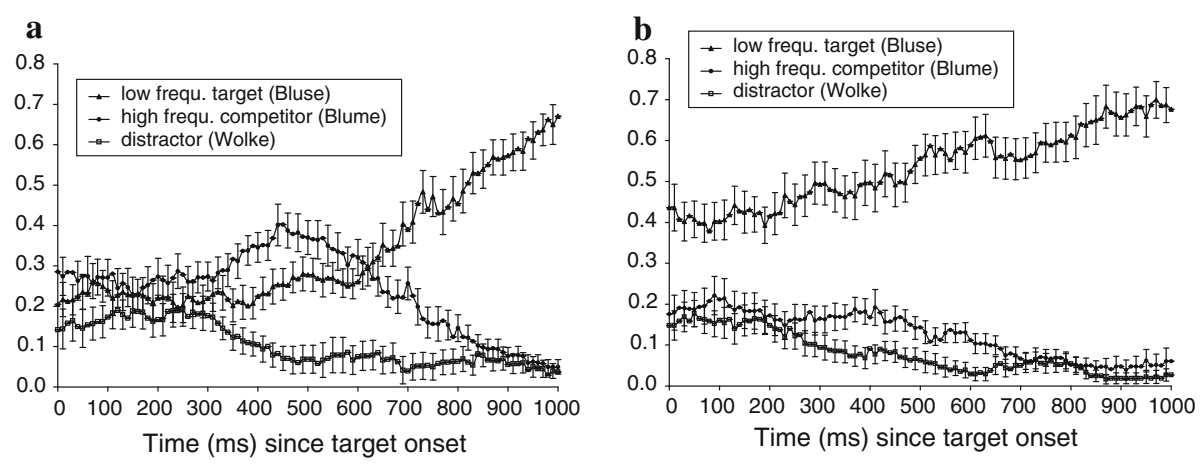

Fig. 3 Fixation proportions over time to target, competitor, and distractor for trials with an unrestrictive verb (a), and for trials with a restrictive verb (b). Error bars represent standard errors of the mean

When the verb was semantically constraining (Fig. 3b), on the other hand, most looks were directed towards the semantically fitting low-frequency target Bluse 'blouse' already at target word onset (40\% for target, $18 \%$ for phonological competitor), and this clear preference for the target was maintained during the following $1000 \mathrm{~ms}$. Thus, based on the semantic information of the verb, listeners could clearly anticipate the upcoming argument of the sentence correctly. Between 200 and $700 \mathrm{~ms}$, listeners also fixated the high-frequency competitor more than the distractor, even though the competitor was semantically implausible. Seemingly, the competitor was still considered a possible word candidate after having heard the restrictive verb, albeit a weaker one than in sentences with unrestrictive verbs.

To statistically analyse the activation of implausible competitors, we calculated the difference values between competitor and distractor in restrictive sentences for two time windows: one extending from 0 to $200 \mathrm{~ms}$ after target onset and a second window from 200 to $700 \mathrm{~ms}$. The duration of the later time window corresponds roughly to the average duration of target words in Experiment 2 (550 ms for target word in restrictive sentences and $564 \mathrm{~ms}$ for target words in unrestrictive sentences). We then compared these difference values against zero in one-sample $t$ tests. If activation of implausible competitors was reduced but not eliminated after restrictive verbs, difference values should differ significantly from zero. Indeed, $t$ tests showed a significant effect $\left(t_{1}[23]=3.38, p<.004 ; t_{2}[19]=2.64, p<.02\right)$ between 200 and $700 \mathrm{~ms}$. In the plausibility rating of restrictive sentences, two sentences with high-frequency competitors (Schublade and Lampe) were rated considerably more plausible than the rest of the sentences. It is possible therefore that the observed activation of competitors in Experiment 2 was enhanced by these two sentences in which the competitors were in fact not strongly implausible. However, one-sample $t$ tests with the difference values between competitor and distractor were still significant when these two sentences were excluded from the analysis $\left(t_{2}[17]=2.39, p<.03\right)$. No significant effect was found between 0 and $200 \mathrm{~ms}$, when fixations were not yet driven by the acoustic input from the target $\left(t_{1} \& t_{2}<1\right)$.

These analyses suggest that high-frequency competitors in Experiment 2 were significantly activated even when semantic verb information rendered them implausible. This finding is in line with Huettig and Altmann (2007) who found increased visual attention to a picture showing the dominant meaning of a homonym, even when semantic context biased towards the subordinate meaning. Dahan and Tanenhaus (2004), on the other hand, had not found significant activation of candidate words that are semantically inappropriate 
but overlap phonologically with the appropriate target. However, in their study competitors and targets were of equal lexical frequency. Taken together the findings suggest that measurable activation of semantically inappropriate words can be found as long as words are high in (lexical or meaning) frequency. A more detailed account of the possible role of frequency in constraining sentences follows in the "General Discussion".

Next, in order to evaluate the influence selective verb restrictions have on targets and competitors respectively, we directly compared target and competitor fixations in the two types of sentences. In one-way repeated measures ANOVAs we found, between 200 and $700 \mathrm{~ms}$, significantly more looks to the target Bluse 'blouse' in restrictive sentences (57\%) than in unrestrictive sentences $\left(26 \% ; F_{1}[1,23]=28.90, p<.001 ; F_{2}[1,19]=50.33, p<.001\right)$. Vice versa, the competitor Blume 'flower' was fixated less often in restrictive sentences $(15 \%)$ than in unrestrictive sentences $\left(31 \% ; F_{1}[1,23]=16.11, p<.002 ; F_{2}[1,19]=\right.$ $6.08, p<.03$ ). The $200-700 \mathrm{~ms}$ window reflects the time during which the target word was being processed. Effects of semantic constraints during this time thus suggest an immediate influence of context on lexical processing. This finding replicates Dahan and Tanenhaus (2004) who also found an early effect of context on spoken-word recognition in Dutch.

However, whether context helped to recognize the intended target or whether it hindered activation of implausible competitors, cannot be established with the above analysis. More looks to the target necessarily imply fewer looks to the other displayed objects; a context effect on competitors could therefore mainly mirror the increase in looks to the target and not an independent inhibitory effect on competitors. Furthermore, we know that listeners can anticipate upcoming referents in this type of task; the question thus arises whether context effects are really just anticipatory effects or whether there is an actual influence on spokenword recognition as the phonetic-to-lexical mapping takes place. In order to address these questions we compared fixation proportions before and after acoustic information about the target word was available to listeners. Between 0 and $200 \mathrm{~ms}$, we found significantly more looks to the target Bluse 'blouse' in restrictive sentences (44\%) than in unrestrictive sentences $\left(24 \% ; F_{1}[1,23]=12.74, p<.003 ; F_{2}[1,19]=9.07, p<.008\right)$. Anticipatory looks to plausible upcoming referents are in line with previous research (e.g., Altmann and Kamide 1999; Dahan and Tanenhaus 2004; Huettig and Altmann 2007). However, during this early time window, an increase in looks to the target was not reflected by a significant decrease in looks to the competitor $(20 \%$ in restrictive sentences and $27 \%$ in unrestrictive sentences; $F_{1}<1 ; F_{2}[1,19]=1.04, p>.3$ ). Note that more looks to the target in restrictive sentences must mean fewer looks to other displayed objects at the same time. Indeed, in restrictive sentences the competitor, the distractor, and the displayed agent all received numerically somewhat fewer looks than in unrestrictive sentences, but the difference was never significant. The same pattern was found when we increased the duration of the early time window such that it included the article preceding the target noun $(-150$ to $200 \mathrm{~ms}$; target fixations: $F_{1}[1,23]=14.65, p<.002 ; F_{2}[1,19]=9.58, p<.007$; competitor fixations: $\left.F_{1}[1,23]=2.48, p>.1 ; F_{2}[1,19]=1.84, p>.1\right)$. The fact that we found in the early time windows a semantic context effect for target pictures, in the absence of an effect for competitors, suggest that in the later time window a context effect for competitors is very likely more than just a reflection of the increase in looks to the target. Rather context seems to have had an independent influence on the activation of competitor words during lexical processing. Taken together, the results suggest that semantic context can help to anticipate upcoming referents, but independently thereof it also modulates the competition process during spoken-word recognition. 


\section{General Discussion}

We present the results from two eye-tracking studies in German that investigate the role of lexical frequency and semantic context constraints in spoken-word recognition. In Experiment 1, German listeners were told to click on either the low frequency object Bluse, 'blouse', or the high frequency object Blume, 'flower'. Beyond the finding that both object names were competing for recognition due to their onset overlap, high-frequency competitors were looked at more often than low frequency competitors. This advantage for the recognition of German high-frequency words is consistent with compelling evidence for a frequency advantage from a variety of paradigms (Connine et al. 1990; Dahan et al. 2001; Dahan and Gaskell 2007; Dupoux and Mehler 1990; McQueen 1993).

Having confirmed lexical frequency effects for our German materials in Experiments 1 and 2 set out to investigate the influence of contextual constraints on the recognition of German nouns. In particular, the influence of selective verb restrictions prior to and during lexical processing was tested. The first finding was that semantic verb information clearly led listeners to anticipate appropriate object arguments: when hearing Die Frau bügelt die Bluse, 'The woman irons the blouse', most fixations were already on Bluse prior to the onset of the object argument. This finding is in line with a number of eye-tracking studies that have found that sentential context can allow the anticipation of upcoming referents in sentences (e.g., Altmann and Kamide 1999, 2007; Huettig and Altmann 2004, 2007; Kamide et al. 2003). However, at a point in time when eye movements reflect the acoustic processing of the target word Bluse, we also found activation of the contextually inappropriate competitor Blume, but the activation of the competitor was weaker in constraining sentences than in unconstraining sentences. This important second finding reveals limitations of contextual constraints on selective lexical activation. While it confirms an immediate influence of context on the activation of phonologically overlapping words (see Dahan and Tanenhaus 2004), it extends previous findings, in that it shows contextual effects are not exclusive in nature. Not fully excluding inappropriate competitors from consideration can help listeners of course to recover from wrong anticipations or misunderstandings. In everyday conversations, sentences can take quite unexpected turns, or seemingly inappropriate verb arguments can become appropriate in further context. For instance, Die Frau bügelt die Blume..., 'the woman irons the flower. ..', might be completed with ... applikation auf ihrem Kleid, '. . . application on her dress'. If Blume were not initially activated in this case, word recognition would probably be seriously delayed. The fact that activation of semantically inappropriate competitors was still observed also verifies the importance of acoustic-phonetic information for lexical competition, and speaks against a selective access view in which only contextually appropriate word candidates are considered for recognition (e.g., Connine et al. 1994; Marslen-Wilson 1989; Miller and Eimas 1995). The results, however, also present a challenge to multiple access models that assume no effect of context on lexical activation (e.g., Massaro 1996; Gaskell and Marslen-Wilson 2002), because context did reduce the activation of semantically implausible competitors significantly.

In principle, two explanations are possible for the fact that high-frequency competitors were activated in constraining sentence contexts in Experiment 2. The first involves a tradeoff between the two factors lexical frequency and semantic context in which frequency can overrule contextual restrictions. As long as a phonological competitor is higher in lexical frequency than a target, the competitor gets activated even when semantics renders it inappropriate; inappropriate competitors that are equal or lower in lexical frequency than the target would not get activated according to this view. On this account, Dahan and Tanenhaus (2004) found no activation of contextually inappropriate competitors because their 
competitors were of the latter type (i.e., as frequent as the target). Frequency would set a threshold for considering lexical candidates during spoken-word recognition, despite contextual evidence against them. However, if lexical frequency can overrule contextual constraints, it is not obvious why high-frequency competitors in Experiment 2 were fixated less often in inappropriate contexts than in neutral contexts. It seems more plausible that contextual information was not overruled in Experiment 2 but rather that context was used to downgrade the activation of inappropriate competitors, while the high lexical frequency enabled competitor activation to be observed in eye movements nonetheless. On this second account, phonological competitors are activated, even when they are inappropriate in a sentence context, but in constraining contexts they are less strongly activated than in unconstraining contexts. On this account, the lack of inappropriate competitor activation in Dahan and Tanenhaus (2004) was due to insufficient sensitivity of the eye-tracking paradigm to observe the activation of competitors that are both implausible and only equal in lexical frequency with their target.

Support for this interpretation also comes from recent eye-tracking studies that tested words with multiple meanings. Huettig and Altmann (2004, 2007) found convincing evidence for the activation of the dominant meaning of an ambiguous word like pen in a context that favours the subordinate meaning (e.g., "The welder locked up carefully, but then he checked the pen and suspected that it was damaged"). Consistent with our results, the object depicting the subordinate meaning of pen (i.e., a cage-like enclosure) received most looks at the onset of "pen" in these biasing sentences, while the object depicting the dominant meaning (i.e., a writing instrument) was only as interesting as the distractors; but at word offset, the object with the dominant meaning received significantly more looks than the distractors. While words with multiple meanings are fully ambiguous, the ambiguity in the present study was only temporal, based on phonemic onset overlap. Therefore in our study, at word offset, activation of inappropriate high-frequency competitors was not found because acoustic information no longer matched with the competitor, but we did find activation of inappropriate competitors while they were acoustically still overlapping with the target.

Theories of spoken-word recognition are generally concerned with the processes by which acoustic-phonetic information is mapped onto stored lexical representations. When interpreting the influence of semantic context on spoken-word recognition, it therefore seems important to distinguish any influence context exerts before the actual word is being heard from an influence of context while the word is being heard. Anticipatory eye movements show that listeners quickly begin constructing detailed interpretations of what they hear, and even initiate hypotheses or expectations about what is likely to follow in a sentence. Research by Kamide et al. (2003) and Kamide et al. (2003), suggests that this anticipation of verb arguments reflects a higher-level process of semantic interpretation which makes semantically fitting verb arguments more readily available (and is thus more than pure lexical priming between verbs and their arguments). But is a semantically inappropriate competitor a weaker competitor during spoken-word recognition because its activation levels are influenced by context or is its weakness just a reflection of the target word being predictable in context? The present data suggest that a weaker competitor is not just the reflection of anticipating the appropriate target. If the decrease in fixations to the competitor during the target word was only due to an increase in fixations to the target, then the same decrease for the competitor should have been observed at a point in time when the target word was not being heard yet (but was anticipated). This was not the case. While more looks to the semantically appropriate target were found before and during processing of the target word, the competitor was only influenced by contextual fit once the target word was being heard. This suggests that semantic context led indeed to a lower activation level for the inappropriate competitor during spoken-word recognition. Thus, the mechanism underlying semantic context effects 
in spoken-word recognition involves at least an inhibitory influence on candidate words that are phonologically similar but not semantically similar to the target word. Since context influenced target fixations prior to lexical processing as well as during lexical processing in Experiment 2, we cannot establish an independent facilitatory effect of context on target activation with our results. But when Dahan and Tanenhaus (2004) analysed a subset of their restrictive sentences for which there was no strong anticipation of the target, they nevertheless found a weak effect of context on target fixations while the target word was being heard. Thus, possibly semantic context also facilitates the recognition of the target word, but this could not be confirmed in the present study in which selective verb information clearly allowed anticipatory responses for the verb argument.

The main goal of the current study was to investigate the nature of the effect of semantic constraints on lexical activation. The debate about the existence and the timing of such constraints is time-honoured, with empirical evidence pointing in different directions. Results from the present studies are generally in line with evidence in support of an early effect of contextual constraints and importantly can show an effect of contextual constraints on spoken-word recognition that is independent of anticipatory responses to expected upcoming target words. Inhibition of semantically ill-fitting competitors was found to be the underlying mechanism for semantic constraint effects with the effect of reducing activation of ill-fitting competitors but not excluding them from competition.

Acknowledgments The experiments were completed while the first author was a postdoctoral fellow at Saarland University. The research was funded by SFB 378 'ALPHA' to the first and second author, awarded by the German Research Foundation DFG. Preliminary results of Experiment 1 and parts of Experiment 2 were presented at the CUNY conference in 2006.

\section{Appendix}

See Table 1.

Table 1 Experimental stimuli used in Experiments 1 and 2, with English translations

\begin{tabular}{llll}
\hline Unrestrictive verb & Restrictive verb & Low frequency target & High frequency competitor \\
\hline Finden & Lichten & Anker (0.48) & Anzug (1.26) \\
'To find' & 'To hoist' & 'Anchor' & 'Suit' \\
Finden & Bügeln & Bluse (0.78) & Blume (1.51) \\
'To find' & 'To iron' & 'Blouse' & 'Flower' \\
Kontrollieren & Reparieren & Bohrer $(0.30)$ & Boxer (0.90) \\
'To examine' & 'To repair' & 'Drill' & 'Boxer' \\
Betrachten & Entzünden & Fackel (0) & Falle (1.52) \\
'To observe' & 'To ignite' & 'Torch' & 'Snare' \\
Kaufen & Spielen & Fagott (0) & Fahrrad (1.26) \\
'To buy' & 'To play' & 'Bassoon' & 'Bicycle' \\
Entdecken & Zerreissen & Flagge (1.11) & Flasche (1.56) \\
'To discover' & 'To tear' & 'Flag' & 'Bottle' \\
Sehen & Essen & Kirsche (0.48) & Kirche (2.33) \\
'To see' & 'To eat' & 'Cherry' & 'Church' \\
\hline
\end{tabular}


Table 1 continued

\begin{tabular}{|c|c|c|c|}
\hline Unrestrictive verb & Restrictive verb & Low frequency target & High frequency competitor \\
\hline Prüfen & Werfen & Lanze $(0.70)$ & Lampe (1.85) \\
\hline 'To test' & 'To throw' & 'Lance' & 'Lamp' \\
\hline Sehen & Pflücken & Mohn (0) & Mond (1.89) \\
\hline 'To see’ & 'To pluck' & 'Poppy seed' & 'Moon’ \\
\hline Erblicken & Erschlagen & Mücke (0) & Mütze (1.04) \\
\hline 'To see' & ‘To slay' & 'Mosquito' & 'Cap’ \\
\hline Bemerken & Erschiessen & Panther (0) & Panzer (1.26) \\
\hline 'To notice' & ‘To shoot/kill' & 'Panther' & 'Tank' \\
\hline Erblicken & Zerstechen & Reifen (0.78) & Reiter (1.38) \\
\hline ‘To see' & 'To burst & 'Tire' & 'Equestrian' \\
\hline Finden & Schneiden & Schilf (0.70) & Schiff (1.99) \\
\hline 'To find' & 'To cut' & 'Reed' & 'Ship’ \\
\hline Sehen & Schieben & Schubkarre $(0)$ & Schublade (0.78) \\
\hline 'To see' & 'To push' & 'Wheelbarrow' & 'Drawer' \\
\hline Sehen & Drehen & Spindel (0) & Spinne $(0.78)$ \\
\hline 'To see' & 'To spin’ & 'Spindle' & 'Spider' \\
\hline Suchen & Ziehen & Stecker $(0)$ & Stempel (0.90) \\
\hline 'To search for' & 'To pull’ & 'Plug' & 'Postmark' \\
\hline Verkaufen & Zerbrechen & Tasse (1.00) & Tasche (1.56) \\
\hline 'To sell' & 'To break' & ‘Cup’ & 'Bag' \\
\hline Erkennen & Besuchen & Tempel (1.04) & Teppich (1.38) \\
\hline 'To recognize' & 'To visit' & 'Temple' & 'Carpet' \\
\hline Mustern & Schütteln & Thermometer $(0.30)$ & Telefon (2.28) \\
\hline 'To examine' & 'To shake' & 'Thermometer' & 'Telephone' \\
\hline Entdecken & Verscheuchen & Wespe $(0.30)$ & Weste (1.49) \\
\hline 'To discover' & ‘To shoo away' & 'Wasp' & 'Vest' \\
\hline
\end{tabular}

In brackets, lexical frequency is reported on a logarithmic scale. (A value of 0 implies that a word occurs less than 9 times in the complete German corpus used in CELEX)

\section{References}

Allopenna, P., Magnuson, J., \& Tanenhaus, M. (1998). Tracking the time course of spoken word recognition using eye movements: Evidence for continuous mapping models. Journal of Memory and Language, 38, 419-439.

Altmann, G., \& Kamide, Y. (1999). Incremental interpretation at verbs: Restricting the domain of subsequent reference. Cognition, 73, 247-264.

Altmann, G., \& Kamide, Y. (2007). The real-time mediation of visual attention by language and world knowledge: Linking anticipatory (and other) eye movements to linguistic processing. Journal of Memory and Language, 57, 502-518.

Baayen, H., Piepenbrock, R., \& van Rijn, H. (1993). The CELEX lexical database (CD-ROM). Philadelphia: Linguistic Data Consortium, University of Pennsylvania.

Barr, D. J. (2008). Pragmatic expectations and linguistic evidence: Listeners anticipate but do not integrate common ground. Cognition, 109, 18-40.

Blutner, R., \& Sommer, R. (1988). Sentence processing and lexical access: The influence of the focusidentifying task. Journal of Memory and Language, 27, 359-367. 
Bonin, P., Barry, C., Meot, A., \& Chalard, M. (2004). The influence of age of acquisition in word reading and other tasks: A never ending story?. Journal of Memory and Language, 50, 456-476.

Connine, C., Blasko, D., \& Wang, J. (1994). Vertical similarity in spoken word recognition: The role of sentence context. Journal of Memory and Language, 32, 193-210.

Connine, C., Mullennix, J., Shernoff, E., \& Yelen, J. (1990). Word familiarity and frequency in visual and auditory word recognition. Journal of Experimental Psychology: Learning, Memory, and Cognition, 16, 1084-1096.

Connine, C., Titone, D., \& Wang, J. (1993). Auditory word recognition: Extrinsic and intrinsic effects of word frequency. Journal of Experimental Psychology: Learning, Memory, and Cognition, 19, 81-94.

Cooper, R. (1974). The control of eye fixation by the meaning of spoken language. Cognitive Psychology, 6, 84-107.

Dahan, D., \& Gaskell, M. (2007). The temporal dynamics of ambiguity resolution: Evidence from spoken-word recognition. Journal of Memory and Language, 57, 483-501.

Dahan, D., Magnuson, J., \& Tanenhaus, M. (2001). Time course of frequency effects in spoken-word recognition: Evidence from eye movements. Cognitive Psychology, 42, 317-367.

Dahan, D., Swingley, D., Tanenhaus, M. K., \& Magnuson, J. S. (2000). Linguistic gender and spoken-word recognition in French. Journal of Memory and Language, 42, 465-480.

Dahan, D., \& Tanenhaus, M. (2004). Continuous mapping from sound to meaning in spoken language comprehension: Immediate effects of verb-based thematic constraints. Journal of Experimental Psychology: Learning, Memory, and Cognition, 30, 498-513.

Duffy, S. A., Morris, R. K., \& Rayner, K. (1988). Lexical ambiguity and fixation times in reading. Journal of Memory and Language, 27, 429-446.

Dupoux, E., \& Mehler, J. (1990). Monitoring the lexicon with normal and compressed speech: Frequency effects and the prelexical code. Journal of Memory and Language, 29, 316-335.

Eberhard, K., Spivey-Knowlton, S., Sedivy, J., \& Tanenhaus, M. (1995). Eye movements as a window into real-time spoken language processing in natural contexts. Journal of Psycholinguistic Research, 24, 409-436.

Fiebach, C. J., Friederici, A. D., Müller, K., von Cramon, D. Y., \& Hernandez, A. E. (2003). Distinct brain responses for early and late learned words. Neuroimage, 19, 1627-1637.

Gaskell, M., \& Marslen-Wilson, W. (1997). Integrating form and meaning: A distributed model of speech perception. Language and Cognitive Processes, 12, 613-656.

Gaskell, M., \& Marslen-Wilson, W. (2002). Representation and competition in the perception of spoken words. Cognitive Psychology, 45, 220-266.

Hallet, P. (1986). Eye movements. In K. Boff, L. Kaufman, \& J. Thomas (Eds.), Handbook of perception and human performance (pp. 10-1-10-112). New York: Wiley.

Huettig, F., \& Altmann, G. (2004). The online processing of ambiguous and unambiguous words in context: Evidence from head-mounted eye-tracking. In M. Carreiras \& C. Clifton (Eds.), The on-line study of sentence comprehension: Eyetracking, ERP and beyond (pp. 187-207). New York, NY: Psychology Press.

Huettig, F., \& Altmann, G. (2007). Visual-shape competition during language-mediated attention is based on lexical input and not modulated by contextual appropriateness. Visual Cognition, 15, 985-1018.

Huettig, F., \& McQueen, J. (2007). The tug of war between phonological, semantic, and shape information in language-mediated visual search. Journal of Memory and Language, 57, 460-482.

Kambe, G., Rayner, K., \& Duffy, S. A. (2001). Global context effects on processing lexically ambiguous words: Evidence from eye fixations. Memory \& Cognition, 29, 363-372.

Kamide, Y., Altmann, G., \& Haywood, S. (2003). The time course of prediction in incremental sentence processing: Evidence from anticipatory eye movements. Journal of Memory and Language, 49, 133-156.

Lucas, M. (1987). Frequency effects on the processing of ambiguous words in sentence contexts. Language and Speech, 30, 25-46.

Luce, P., Goldinger, S., Auer, E., \& Vitevitch, M. (2000). Phonetic priming, neighborhood activation, and PARSYN. Perception \& Psychophysics, 62, 615-625.

Luce, P., \& Pisoni, D. (1998). Recognizing spoken words: The neighborhood activation model. Ear and Hearing, 19, 1-36.

Marslen-Wilson, W. (1989). Access and integration: Projecting sounds onto meaning. In W. Marslen-Wilson (Ed.), Lexical representation and process (pp. 3-24). Cambridge, MA: MIT Press.

Marslen-Wilson, W. (1990). Activation, competition, and frequency in lexical access. In G. T. M. Altmann (Ed.), Cognitive models of speech processing: Psycholinguistic and computational perspectives (pp. 148172). Cambridge, MA: MIT Press. 
Massaro, D. (1996). Integration of multiple sources of information in language processing. In T. Inui \& J. McClelland (Eds.), Attention and performance XVI. Information integration in perception and communication (pp. 397-432). Cambridge, MA: MIT Press.

Matin, E., Shao, K., \& Boff, K. (1993). Saccadic overhead: Information processing time with and without saccades. Perceptual Psychophysics, 53, 372-380.

McClelland, J., \& Elman, J. (1986). The TRACE model of speech perception. Cognitive Psychology, 10, 1-86.

McQueen, J. M. (1993). Rhyme decisions to spoken words and nonwords. Memory \& Cognition, 21, $210-222$.

Miller, J., \& Eimas, P. (1995). Speech perception: From signal to word. Annual Review of Psychology, 46, 467-492.

Moss, H., \& Marslen-Wilson, W. (1993). Access to word meanings during spoken language comprehension: Effects of sentential semantic context. Journal of Experimental Psychology: Learning, Memory \& Cognition, 19, 1254-1276.

Norris, D., Cutler, A., McQueen, J., \& Butterfield, S. (2006). Phonological and conceptual activation in speech comprehension. Cognitive Psychology, 523, 146-153.

Norris, D., \& McQueen, J. (2008). Shortlist B: A Bayesian model of continuous speech recognition. Psychological Review, 115, 357-395.

Oden, G., \& Spira, J. (1983). Influence of context on the activation and selection of ambiguous word senses. Quarterly Journal of Experimental Psychology, 35, 51-64.

Onifer, W., \& Swinney, D. (1981). Accessing lexical ambiguities during sentence comprehension: Effects of frequency of meaning and contextual bias. Memory \& Cognition, 9, 225-236.

Schulte im Walde, S. (2008). Human associations and the choice of features for semantic verb classification. Research on Language and Computation, 6, 79-111.

Seidenberg, M., Tanenhaus, M., Leiman, J., \& Bienkowski, M. (1982). Automatic access of the meanings of ambiguous words in context: Some limitations of knowledge-based processing. Cognitive Psychology, 14, 489-537.

Sereno, S. C., Pacht, J. M., \& Rayner, K. (1992). Effect of meaning frequency on processing lexically ambiguous words. Psychological Science, 3, 296-300.

Simpson, G. (1981). Meaning dominance and semantic context in the processing of lexical ambiguity. Journal of Verbal Learning and Verbal Behavior, 20, 120-136.

Swinney, D. (1979). Lexical access during sentence comprehension: (Re)consideration of context effects. Journal of Verbal Learning and Verbal Behavior, 18, 645-659.

Tabossi, P. (1988). Effects of context on the immediate interpretation of unambiguous nouns. Journal of Experimental Psychology: Learning, Memory, and Cognition, 14, 153-162.

Tabossi, P., Colombo, L., \& Job, R. (1987). Accessing lexical ambiguity: Effects of context and dominance. Psychological Research, 49, 161-167.

Tabossi, P., \& Zardon, F. (1993). Processing ambiguous words in context. Journal of Memory and Language, 32, 359-372.

Tanenhaus, M., \& Donenwerth-Nolan, S. (1984). Syntactic context and lexical access. Quarterly Journal of Experimental Psychology, 36, 649-661.

Tanenhaus, M., Leiman, J., \& Seidenberg, M. (1979). Evidence for multiple stages in the processing of ambiguous words in syntactic contexts. Journal of Verbal Learning and Verbal Behavior, 18, 427-440.

Vitevitch, M. (2002). Influence of onset density on spoken-word recognition. Journal of Experimental Psychology: Human Perception and Performance, 28, 270-278.

Vitevitch, M., \& Luce, P. (1998). When words compete: Levels of processing in spoken word recognition. Psychological Science, 9, 325-329.

Vitevitch, M., \& Luce, P. (1999). Probabilistic phonotactics and neighborhood activation in spoken word recognition. Journal of Memory and Language, 40, 374-408.

Weber, A., \& Cutler, A. (2004). Lexical competition in non-native spoken-word recognition. Journal of Memory and Language, 50, 1-25.

Weber, A., Melinger, A. (2008). Name dominance in spoken word recognition is (not) modulated by expectations: evidence from synonyms. In Proceedings of the international speech communication association workshop: Experimental linguistics (pp. 205-208). Athens, Greece.

Whitney, P., McKay, T., Kellas, G., \& Emerson, W. (1985). Semantic activation of noun concepts in context. Journal of Experimental Psychology: Learning, Memory, and Cognition, 11, 126-135.

Zwitserlood, P. (1989). The locus of the effects of sentential-semantic context in spoken-word processing. Cognition, 32, 25-64.

Zwitserlood, P., \& Schriefers, H. (1995). Effects of sensory information and processing time in spoken-word recognition. Language and Cognitive Processes, 10, 121-136. 